\title{
The Shall Design Test Development Model for Hardware Systems
}

\author{
Mike Heuchling, Wolfgang Ecker, Michael Mrva \\ Siemens AG, ZT ME 5, 81730 Munich, Germany \\ Tel. 0049 - 89 - 63653359 \\ Fax 0049 - 89 - 63644950 \\ Mike.Heuchling@mchp.siemens.de \\ Wolfgang.Ecker@mchp.siemens.de \\ Michael.Mrva@mchp.siemens.de
}

\begin{abstract}
The object of this paper is to present a development model which helps to provide a clearer organizational structure for new designs by linking the separate aspects of a development process (analysis, design, testing). Particular emphasis is placed on the interrelation between problem analysis ("Shall Model") and implementation ("Design Model"), and also between problem analysis ("Shall Model") and testing procedure ("Test Model"). The consequences of redesigns as well as the integration of reusable designs in new designs are also discussed with reference to use of the Sdevelopment model.
\end{abstract}

\section{Keywords}

SDT development model, design complexity, design method, unified method, requirements, model linking, reuse, generalization concepts, pattern

\section{INTRODUCTION}

\subsection{Design problem}

It is common knowledge among experts that the complexity of ICs expressed in terms of gates per chip, wiring length, gate density etc. will increase dramatically in the coming years (SIA 1995), (Preis 1996). The question that this poses is how to minimize the growing resource requirement involved in the manufacture of such ICs by means of new specification/design methodologies. Moreover, the continuously expanding functional scope of integrated circuits has a direct influence on the resource requirements involved in proving that the circuit is free from errors (verification) and in proving that the implemented functionality matches the specified functionality for the circuit (validation). The following diagram (Figure 1) (VIUF 1996) thus indicates that, taking as a basis a circuit of a given gate size, significantly more lines of code 
(LoC) are required in order to describe the corresponding simulation model than are required to describe the circuit itself (unit under test).

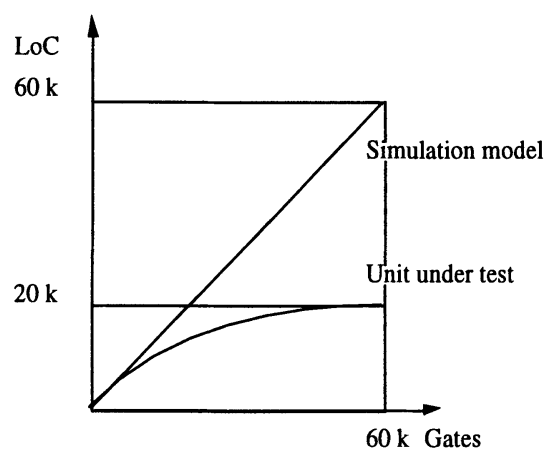

Figure 1 Design complexity vs. simulation complexity

Consequently, the growing functional scope of integrated circuits results not only in a specification and design problem but also, principally, in a testing problem. Many publications deal with verification and validation techniques as topics separate from the problems of design. The question as to how the testing procedure relates to the demands made on a product or how such tests can be systematically derived from given specifications is usually ignored.

\subsection{Development models}

In the analysis of existing specification/design methods (e.g. SADT (Davis 1990), HRT-HOOD (Burns 1995) or in the generation of simulation models from natural language specifications (Cyre 1996)) it is apparent that the majority of these models scarcely take account of the testing problem and totally ignore the documentation problem, the basic problem affecting all questions of reuse. The waterfall and spiral models from Boehm along with the V model (including VP, VR and X models) from Hodgson are described in the following with regard to their intrinsic advantages and disadvantages.

Waterfall model (Boehm 1980) An important advantage of the waterfall model is the clear distinction between analysis stage, design stage and implementation stage, where precisely one validation/verification process is assigned to each stage. Since only finished documents/code etc. are passed between the individual stages, what is referred to as "information tracing" (what has changed, when, how and why?) is not possible. A further disadvantage of the waterfall model is that the dynamics of the design process (e.g. due to error detection, product modification etc.) are taken into 
consideration only by complete backtracking of the design along the development procedure.

Spiral model (Boehm 1986 (August)) The principal new feature here is the consideration of recurring activities in the development process such as analysis, design and testing, which occur correspondingly in each development step. Here, in similar fashion to the waterfall model, the cycles for each development step are detached from the cycles in the following development step. However, the spiral model does not take into consideration either the onward utilization of acquired knowledge or the reuse of designs.

V model (including VP, VR and X models) (Hodgson 1991) This model encompasses a significant quantity of interesting proposals. Hodgson actually breaks down the development process into a partitioning phase and an integration phase. The partitioning phase deals with the design of a system through partitioning of the problem. This phase, in similar fashion to the waterfall model, again contains corresponding analysis and design stages. Each partitioning stage, which defines the manner in which the design is split up, is assigned an integration stage. During the integration phase, the subdesigns which have now resulted from partitioning are linked with one another (integrated) in accordance with the organizational division of the design. Testing of the units defined by the partitioning process subsequently takes place at each integration stage. The prototype concept finds expression in the VP model in the fact that each partitioning/integration stage is regarded as constituting prototype implementation. The VR model (and also the $\mathrm{X}$ model) expands the $\mathrm{V}$ model to the effect that each result of each partitioning stage and also each integration stage is stored in a library assigned to the stage in question. It must be acknowledged here as a disadvantage that tests are performed not immediately but exclusively only after the integration has taken place. Consequently, system errors can also only be detected following system integration. A further disadvantage lies in the fact that there is no interrelation between the reuse libraries (see the $\mathrm{X}$ model).

\subsection{Conclusions}

The above analysis indicates that a model is required which incorporates the advantageous features of the aforementioned models and eliminates the disadvantageous aspects. In other words, there is a need for a model which

- realizes a gradation of the analysis/design/implementation stages,

- takes into consideration recurring activities in the development process,

- links the development steps to their recurring activities by means of a corresponding information model, and also

- defines specifically how to deal with errors and with changes in the development process, 
- enables the reuse of knowledge, solutions etc. in their own context,

- defines tests to be performed as early as the partitioning stage, - gives consideration to the documentation.

\section{THE SDT PARTITIONING PROCESS}

By analogy with the differentiation of the development process into a partitioning phase and an integration phase, the Shall Design Test development model (SDT development model for short) is divided into the SDT partitioning process and the SDT integration process. The aspect of the reuse of designs is discussed in the third main topic below, "The SDT reuse process".

\subsection{Shalls}

Shalls vs. requirements If we look at the product development process (customer analyst designer technologist ...), then the "requirements" are considered to be the demands and stipulations formulated by the customer in the form of a requirements specification (in accordance with (IEEE 1993), for example).

"Shalls" should then be understood as being an extension to the above requirement concept, and should describe general requirements in terms of "What the product shall do, have ...". In other words, shalls additionally contain input specified by the analyst, the designer, the technologist etc. The descriptive form employed may be informal or formal descriptions, and also diagrams, sketches, illustrations etc. If we look at the above process chain, then shalls can be organized in accordance with the chain of product development. But the following forms of organization are also possible:

- from a software design viewpoint, on the basis of a design methodology (Shlear 1996), (Booch 1996), (Coleman 1993), (Jacobson 1995) use case $\rightarrow$ object model $\rightarrow$ object message diagram $\rightarrow \ldots$, or

- from a hardware design viewpoint, on the basis of a time abstraction (Ecker 1995), (Gajski 1994), (Hein 1996)

purely functional $\rightarrow$ system event $\rightarrow$ instruction cycle $\rightarrow$ clock cycle $\rightarrow$ gate propagation.

In any case, shalls do not constitute any mapping of the problem onto the design domain, description of the solution in VHDL or Ada (Bauer 1996), or any mapping onto the test domain, such as the description of tests by using a test specification language for example.

Shalls $v s$. analysis Shalls not only emphasize the problem (problem definition, problem delimitation), they also incorporate conceptional solutions at the relevant 
abstraction level (problem comprehension at the relevant abstraction level). Accordingly, shalls are further conceived as an analytical form of problem registration, they incorporate solution aspects. Solution aspects make the problem handleable and lead to further subproblems. In this respect, each subsolution (actual-state example, note) along with each problem description forms part of a problem perception.

\subsection{Designs}

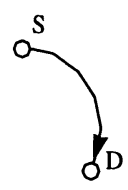

Figure 2 Translation of shalls into designs

While S (set of shalls) specifies the problem or the solution, e.g. by means of ESCs (Kahlert 1993), Petri networks (Camurati 1993), mathematical functions etc., D (design) describes its translation into practice (Waxman 1995). This translation is performed with the aid of a programming language (such as Ada) or a hardware description language (such as VHDL) which supports the description of the concepts used or described in the shall. The realization of these concepts can be handled manually (by programming) on the one hand, but also automatically (by generation) (Coleman 1993), (Fritzsch 1995) on the other. D should be understood as a prototype in the result.

\subsection{Tests}
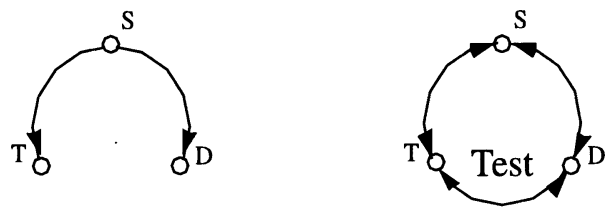

Figure 3 Translation of shalls into tests

The object of T (test) is to check D with regard to its functional and nonfunctional identity with the idea $\mathrm{S}$ (ensuring that performance, behavior, size etc. conform). The 
checks can be performed, for example, by generating I/O patterns, code review or data path analysis. (Boehm 1984) designates and classifies the verification/validation procedures as follows:

\begin{tabular}{cll}
\hline techniques & manual & automated \\
\hline simple & reading & $\begin{array}{l}\text { automated cross referencing } \\
\text { manual cross referencing } \\
\text { simple automated models }\end{array}$ \\
& interviews & \\
& checklists & \\
& manual models & \\
& simple scenarios & \\
\hline detailed & detailed scenarios & detailed automated models \\
& mathematical proofs & prototypes \\
\hline
\end{tabular}

Within the context of automatic checking, tracing and scenario model creation (i.e. the generation of in-sequences and out-(should-be-)sequences; evaluation of the should-be sequence with reference to the actual sequence) play a major role. The creation of tests from shalls can, in similar fashion to the design case, be handled manually (by definition) on the one hand, but also automatically (by generation) (Vemuri 1995), (Sahraoui 1995) on the other.

\subsection{Error handling during a partitioning step}

If $\mathrm{T}$ contains a scenario model, then the interaction between $\mathrm{T}$ and $\mathrm{D}$ is a simulation. If an error occurs during the application of $\mathrm{T}$ on $\mathrm{D}$, this can mean that:

- the design is errored because $\mathrm{D}$ is inconsistent with regard to $\mathrm{S}$ (modification of D);

- the tests are errored because $\mathrm{T}$ is inconsistent with regard to $\mathrm{S}$ (modification of T);

- the shalls are errored because $S$ is incomplete or contradictory (modification of S);

This modification continues to take place until S, D and T are made consistent with one another. The question as to how much $\mathrm{S}$ specifies, $\mathrm{D}$ correspondingly realizes and $T$ examines depends substantially on the granularity of the partitioning process to be presented in the following. If we assume that in the first step $S$ merely specifies just "use cases" (Jacobson 1995), then D could contain the names of the actors and the objects as well as their relations and T could check this name/relation consistency. 


\subsection{The SDT development stages}
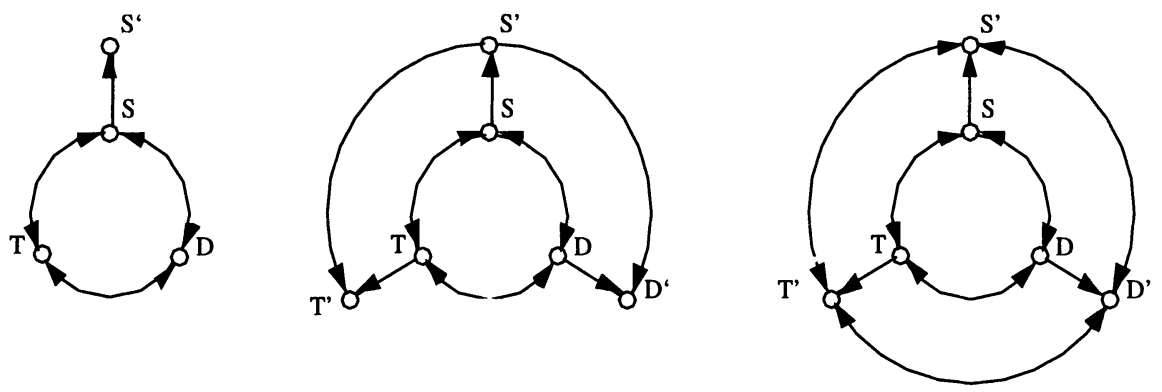

Figure 4 The SDT development stages (individually)

When S, D and T have been made consistent with one another, then $\mathrm{S}$ can be refined. This refinement should be understood as providing detail for the problem or as viewing the same problem on a lower abstract level. As an example in software terms, $S$ could be the consideration of the problem on the basis of "use cases" (application scenarios), whereas S" contains the definition of objects (via CRC cards (Beck 1993)) which realize individual "use cases" through cooperation. In other words, $S$ " does not generally represent a functional extension to $S$, but rather provides detail for the problem. In similar fashion to the relationship between $S^{\prime}$ and $S, D^{\prime}$ is a refinement of $\mathrm{D}$. We are dealing here with the consideration of subfunctionalities which arise from partitioning of the problem. $D^{\prime}$ accordingly specifies a set of subcomponents which are reused in a different way in $\mathrm{D}$. The manner in which these subcomponents

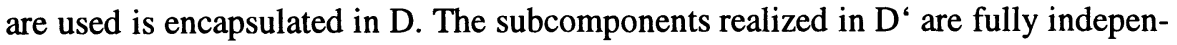
dent of one another. On the one hand, $\mathrm{T}^{\prime}$ provides all the tests which derive from the

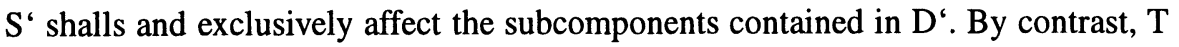
contains only those tests which are required for the checking of $\mathrm{S}$.

This process of refinement can be expanded as required and can thus be applied to any desired development concepts (e.g. hardware development with design at system level $\rightarrow$ algorithmic level $\rightarrow$ RT level $\rightarrow$... (Siegel 1990)).

Each development stage is associated with its following stage. Shalls imply further shalls on a higher abstraction level, designs are partitioned with regard to an architecture into components which require concepts of a low abstraction level for their realization, and tests are dependent on one another both in the context of the shalls and also in the context of the design. Essential to the SDT development model is the "shall"-centered view of the development of a product. Shalls describe the "what is to be realized" (design) and "what is to be tested" (test) respectively. The design and the test are determined by the shalls. In the context of the reuse process to be discussed later, access to a realization, possibly just one component of a design, is effected by way of the description assigned to the component (integrated and optimized shall model). 


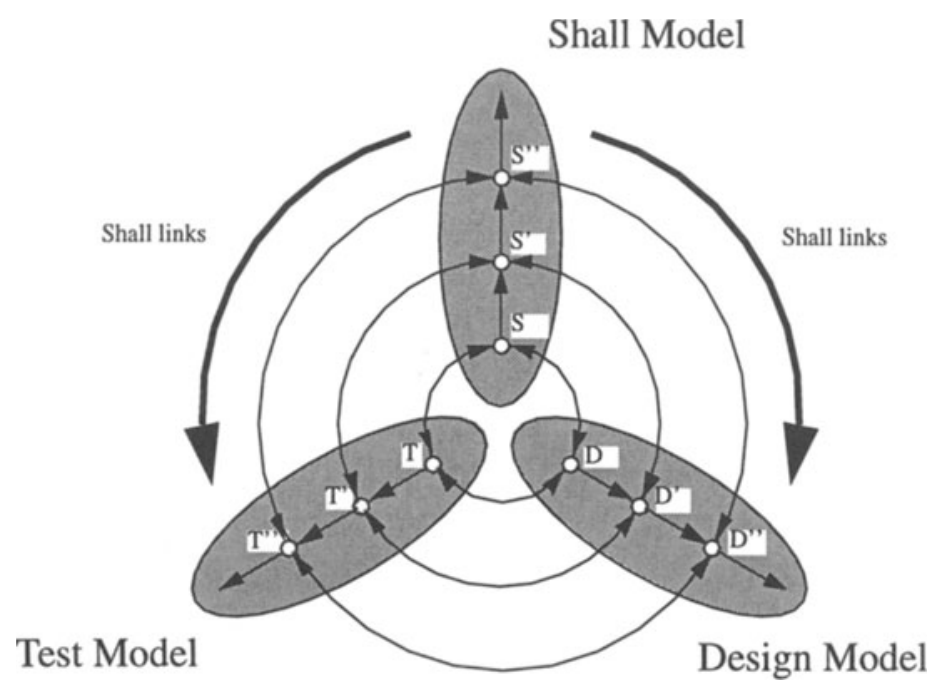

Figure 5 The SDT development stages

\subsection{The SDT models with their relations}

The Shall Model When we consider a set of shalls together with their relations, then we speak of a Shall Model. The Shall Model represents an information model which interconnects the shalls across the abstraction levels. If attributes such as author, reason for the shall definition, priority, creation date etc. are added to the shalls, then specialist decisions to be made in the future can be coordinated with already existing decisions, for example through the medium of consultations or through contextual knowledge.

\section{Shall Model}

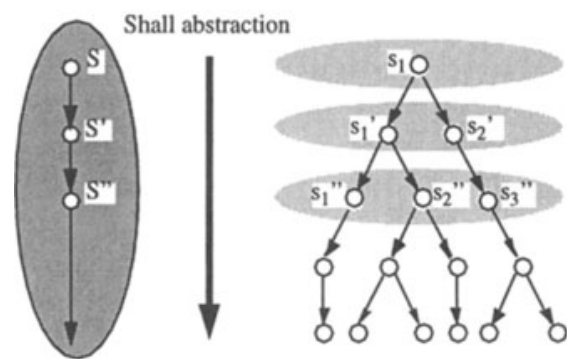

Figure 6 The Shall Model 
In general, shalls of a lower abstraction level (such as $s 1^{\prime}, \mathrm{s} 2$ ' for example) have their origin in shalls of a higher abstraction level (such as s1 for example). The 1:n relationship of shalls across the abstraction levels represented in the Figure 6 should be understood as being a special case. As an example demonstrating the opposite situation, let us assume that two shalls $\mathrm{s}^{\prime}$,, $\mathrm{s} 2^{\text {' }}$ are defined which have a relation to a shall s1".

s1": The system should be capable of being reset to a defined status at any time.

s2‘ : The system has as its interface a signal bus (RESET and ENABLE) and a data bus (12-bit).

s1": The RESET signal is an asynchronous signal.

But it is also possible to add special shalls which, although they are abstractionspecific, have no mandatory relation to shalls of a higher abstraction level (autonomous shalls). Primarily, specialist decisions are added which have no equivalent on a higher abstraction level. For example, if the behavior of tasks has been described and hardware resources have been specified, then a shall:

s1“": The tasks are to be distributed in accordance with a task schedule algorithm XYZ.

has no equivalent in the task definition or the hardware resource description. Such specifications originate from the expert knowledge of the author concerning the optimum distribution of processes. The freedom of the designer plays a decisive role here.

The Design Model In similar fashion to the Shall Model, the Design Model contains the set of all designs (subdesigns) which are interrelated across the relevant abstraction levels. The dependencies of the designs represented as a tree structure similar to the Figure 6, should again be understood as being a special case.

It is thus conceivable that one component can contain different subcomponents, but also that a number of components may refer back to one subcomponent (procedure in a library). Moreover, it is also possible that further components may be added which have no equivalent in designs of a higher level but which result from autonomous shalls. A possible example here would be the design of a component which realizes and monitors the distribution of processes. Now repartitioning of the design means that the links between the components of the Design Model are changed, but also that individual component descriptions are modified.

The Test Model Each shall is assigned at least one test on the same abstraction level. This determines how the set of designs having relations to this shall are to be checked regarding their compliance with the specifications defined in the shall. The Test Model interrelates these test procedures via the different abstraction levels.

Tests can be interrelated on the one hand by way of the type of the test procedure and on the other hand by way of the changing interface of a component. If runtime limits are to be monitored or signal sequences examined, for example, then the detailing of the behavior and of the interface of designs should be pursued over a number of abstraction levels. To the extent by which new shalls are added in the 
Shall Model, the tests are likewise independent of tests at superordinate abstraction levels.

Up to now the Shall Model, the Design Model and the Test Model have been considered in separation. Now their dependencies will be explained. The mapping of the Shall Model onto the Design Model will be referred to as SD mapping, and the mapping of the Shall Model onto the Test Model as ST mapping.

SD mapping A relationship between shalls and designs exists only on the same abstraction level. The mapping of shalls onto designs is not generally speaking unambiguous because, on the one hand, a shall can relate to a number of designs and, on the other, a shall can imply a number of design variants.

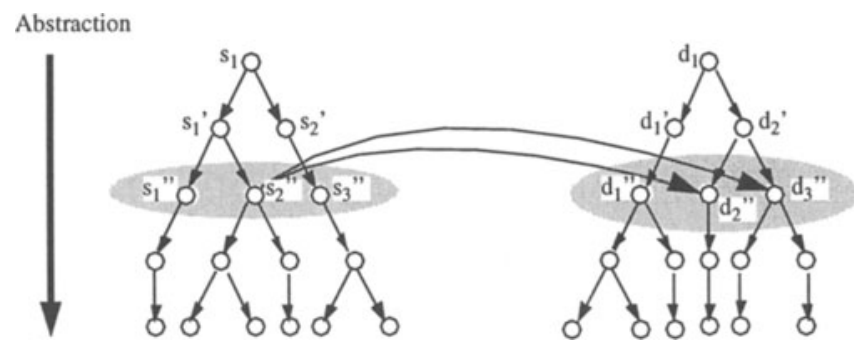

Figure 7 Shall-Design mapping

In any case, each shall has at least one reference to a design from the Design Model. An example of the ambiguity of the mapping of shalls onto designs would be the specification of a standard to be complied with by a set of components from the Design Model.

Mapping in the reverse direction, i.e. the mapping of designs to shalls, is similarly ambiguous. This is because to the same extent that each shall references at least one component of the Design Model, each design has to implement at least one shall.

ST mapping The relationship between the Shall Model and the Test Model exists exclusively between shalls and tests of the same abstraction level. Here too this mapping is ambiguous because a shall which is to be implemented by a number of components has to be checked for each component (component-specific test). In the reverse situation, one test can check a number of shalls for one component. This can be expedient, for example, if it means that a duplication of test cases for a component can be avoided.

Benefits of model creation and model networking The benefit of this type of interlinking of the shalls, designs and tests within the relevant models and beyond the models (via SD mapping and ST mapping respectively) is the capability it offers to 
determine the consequences of changes in the design. If a change occurs in the shalls, for example, then the corresponding shalls in the following abstraction levels can be determined via the Shall Model, and the corresponding designs can be determined by means of appropriate SD mapping. The designs affected on a lower abstraction level can be traced through the Design Model, and can also be traced by tracing the shalls in the Shall Model with their appropriate SD mapping. The same applies by analogy in the case of changes in the Design and Test Models.

Through the provision of the individual models it is possible to trace the information linked within the models and so to manage the implications arising from changes in the development process.

\section{THE SDT INTEGRATION PROCESS}

The principle concern of the SDT partitioning process is to have control over the complexity of the design by means of problem partitioning. The result produced following the last development step (abstraction level) is a set of specifications in the Shall Model, a set of detailed components in the Design Model, and a set of concrete tests for the shall/design pairs in the Test Model. In analogy with the V Model as per Hodgson, the components should be combined (integrated) in accordance with the architectural concept defined at the more abstract level and should be optimized in accordance with efficiency criteria. Similar considerations apply to the Test Model and the Shall Model.

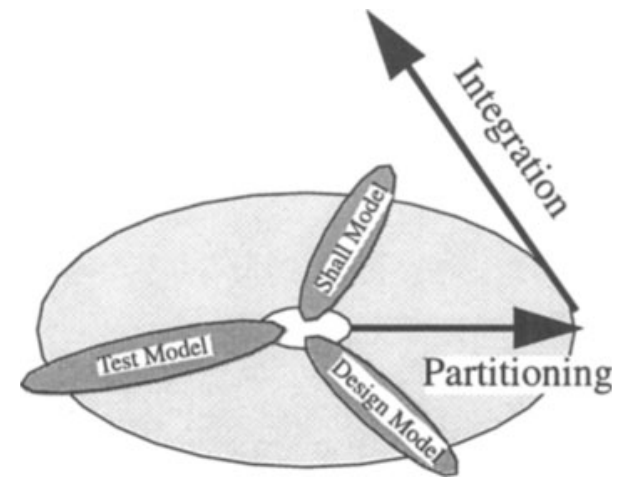

Figure 8 The SDT partitioning and integration process 


\subsection{Design integration}

If the development process has been carried out down to the smallest possible degree of detail, then a set of autonomous prototypes will exist in the Design Model for each abstraction level. If we assume for example that $D$ " represents the smallest possible degree of detail (Figure 9), then these realized concepts can be reorganized (optimized) in accordance with efficiency criteria such as hardware technology, operating system environment etc. Integration of the optimized subconcepts from $D^{\text {“ }}$

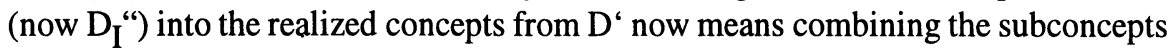
(now implementations) contained in D" in accordance with the partitioning defined

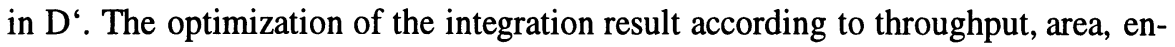
ergy consumption, ... etc again produces implementations $D_{\mathrm{I}}$ ' which constitute the basis for further integrations.

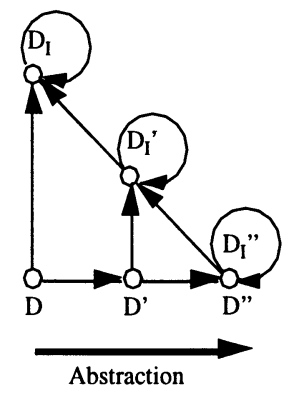

Figure 9 Integration and optimization of the Design Model

$D_{\mathrm{I}}$ is ultimately the final product, provided it satisfies the test procedure to be presented later in this paper.

\subsection{Test integration}

In order to explain the necessity for the integration of tests, it should be emphasized that the complete optimized realization may possibly be required for the checking of shalls (e.g. for performance tests, tests regarding the size of modules, test regarding energy consumption etc.). The integration of tests means that elements of information, such as previously used test cases or partial results from individual tests, are propagated to produce tests on a higher abstraction level. An optimization of tests could be achieved in terms of minimizing resource deployment through the elimination of redundant test situations, for example. 


\subsection{Shall integration}

The combination of the shalls in similar fashion to the integration performed for the Design Model and Test Model is now the combination of all information elements (shalls) which were required for the realization. If this information, which has a defined relationship to its components in the Design Model, is optimized for one target group (customer, designer, ...), the result is appropriate documentation for the referenced components (customer $\rightarrow$ user instructions, designer $\rightarrow$ reuse description, ... etc. ).

\subsection{Error handling during the integration process}

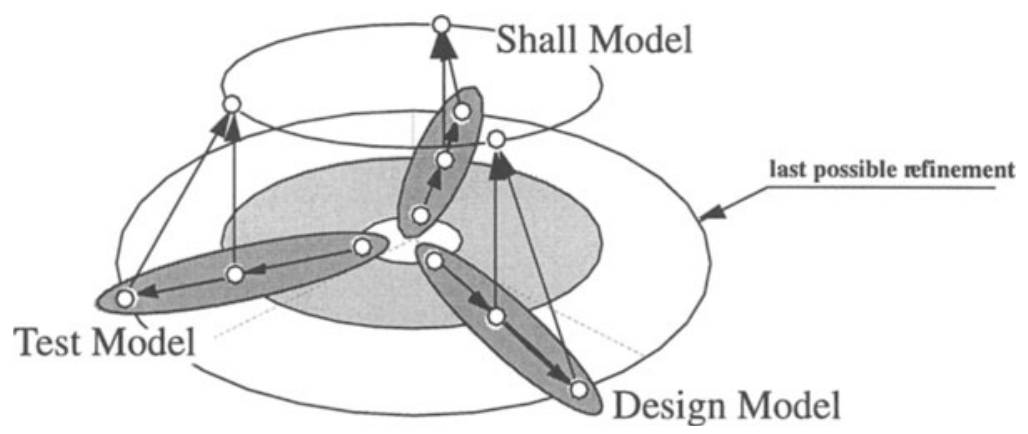

Figure 10 Error handling during the integration process

In the SDT development model, the first integration step can be represented for all three models as per Figure 10. The tests, which can only be realized on the relevant implementation level, can now be performed on this level. If an error occurs during such testing, the question of the source of the error then arises. Either

- the Test Model was not modeled accurately at this level or

- the Design Model was not appropriately realized or

- shalls in the Shall Model imply realizations (tests or designs) which result in errors on a low abstraction level.

If the Test Model is consistent with respect to the Shall Model, then the Design Model must be changed. The components concerned must be determined at the relevant abstraction level before returning to the partitioning process. The concerned shall, design, and test components are then traced, analyzed and changed at this level (only in accordance with the defined paths in the model in question) and are integrated/optimized again in stepwise fashion. 


\subsection{Partial integration}

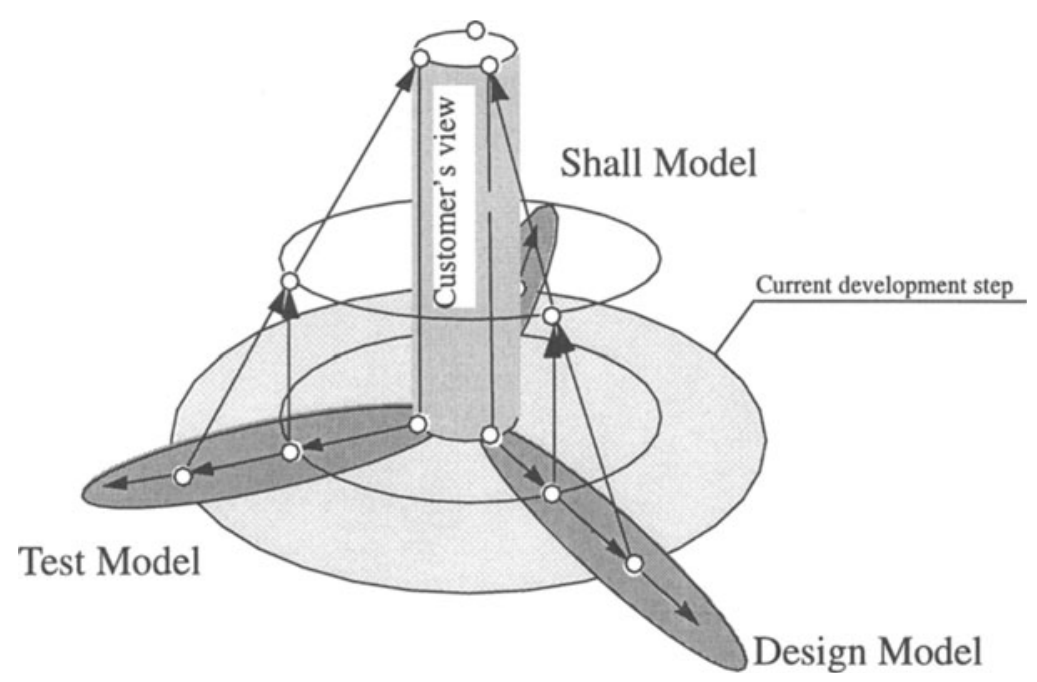

Figure 11 Partial integration during the SDT partitioning process

By means of partial integration it is now possible to check the project status for any desired development step. Starting from the current development step this is made possible

- firstly through the integration of the Design Model (produces the realized design as a virtual prototyp),

- secondly through the integration of the shalls (produces the problem specification as a documentation) and,

- last but not least, through the integration of the Test Model (produces the validation/verification procedures).

If the integration is always performed on the basis of the start shalls (requirements of the customer), then the customer can be informed about every development step, decisions can be discussed and undesirable (from the customer viewpoint) development trends can be avoided at an early stage.

\section{THE SDT REUSE PROCESS}

The last main topic for discussion is reuse. The object of this section is the operation of the SDT development model with reference to aspects of reuse. 
Reuse refers primarily to the reuse of realized components. In order that a component may possess reusable characteristics, the possible field of applications for this component must be known. We speak here of the adaptability/generality of a component within a particular domain (Mrva 1997). This capability can be achieved on the one hand through the general character of a component (plug and play), through appropriate scaling and parameterization options or through partial redesign (e.g. redefinition/extension of methods in the $\mathrm{OO}$ context). The actual work associated with the reuse aspect lies principally in the design of a reusable component. The reuse of a component is the result of the work invested in the design phase. If there is a desire to realize a reusable component, then its adaptability/generality must be determined (functional scope, redundancy, parameterizability etc.) (Flor 1996). With regard to the reusability of a component, efficiency and flexibility are at opposite ends of the spectrum (how flexible vs. efficient should a component be). Whereas efficiency is characterized by the optimized composition of concepts, flexibility requires that concepts be structured in a clear and open manner. Open-structured concepts are employed in the SDT development model in the partitioning process, while integrated/optimized concepts may be found in the integration process.

Since the integration and optimization information is defined during the partitioning process, all project experience relating to a project may be found in the shall, design, and test models and their links. Reuse in this model can take the form of the reuse of project experience gained from any other project on the one hand, or the application of appropriate generalization concepts across a number of projects on the other.

\subsection{Simple-level and multi-level reuse}

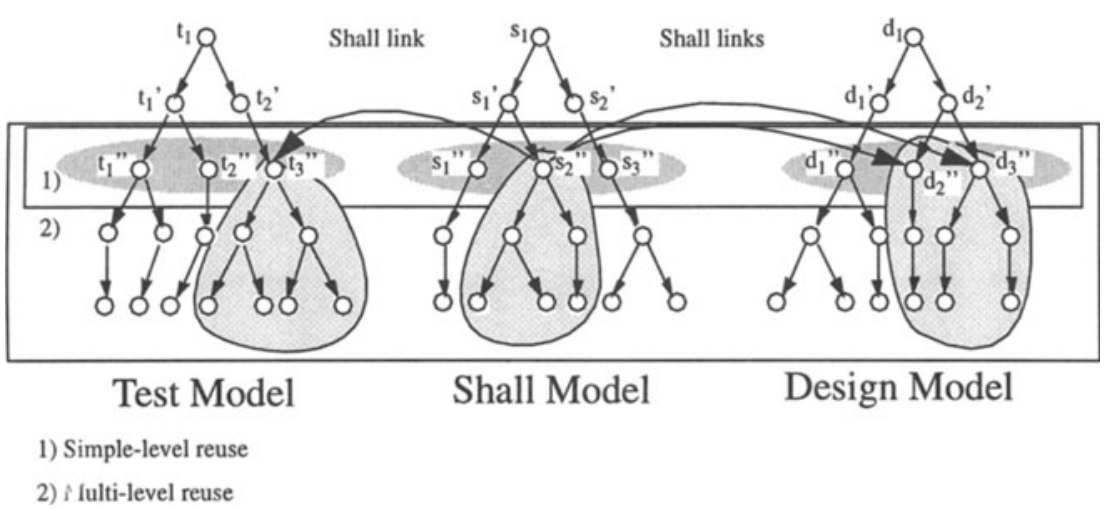

Figure 12 Simple-level reuse vs. multi-level reuse 
Simple-level reuse is understood to consist in the simple use of shalls, designs and tests from any other project. This can take place from one model to another model on the one hand, but also via the shall links from shalls to the Test Model or Design Model as appropriate. Whereas simple-level reuse is exclusively concerned with realizations from the current development step of other projects, multi-level reuse denotes the use of solutions from the current development step through to the last possible development step for a component (Figure 12).

\section{Shall Model}

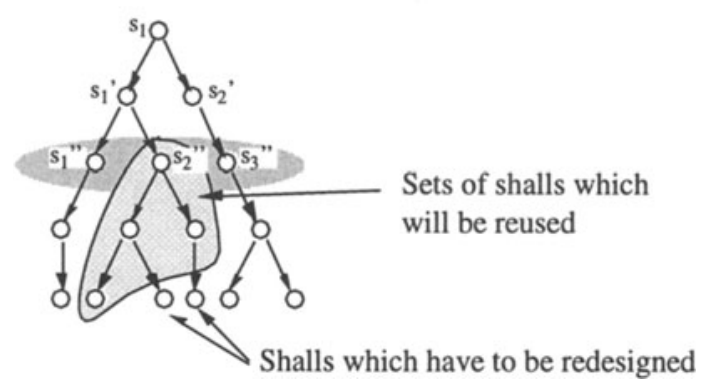

Figure 13 Partial multi-level reuse

With reference to the subject of reuse, partial multi-level reuse means that parts of a realization from a particular abstraction level onwards are redefined (e.g. redefinition/extension of methods in the $\mathrm{OO}$ context). The application of multi-level reuse or partial multi-level reuse offers a look-ahead facility to following design steps with regard to the constraints and realizations determined by the reused component. Later design steps are accordingly characterized by the use of components which have already been realized. Let us take the reuse of a Shall Model as an example of partial multi-level reuse .

\subsection{Generalization concepts}

The real benefits of the reuse capability actually consist in the generalization of solutions across a number of projects. Two situations should basically be differentiated:

- Projects (e.g. project A, B, C) which specify the same problem on one level of abstraction but which refer back to similar solutions on a lower level (Figure 14),

- Projects which specify similar problems on one level of abstraction but which refer back to the same solutions on a lower level (Figure 15).

It would consequently be possible for example in the former case to imagine a component which, by means of a parameter, specifies the sort algorithm to be used 


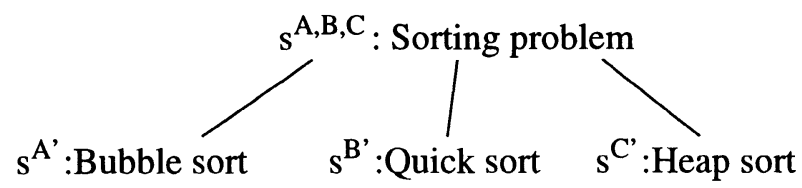

Figure 14 Specification of the same problem with different solutions

$\mathrm{s}^{\mathrm{A}}$ :DB retrieval problem $\quad \mathrm{s}^{\mathrm{B}}$ :Editor problem $\quad \mathrm{s}^{\mathrm{C}}$ :Sorting problem

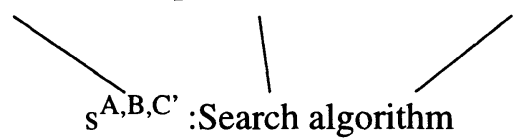

Figure 15 Specification of "different" problems with the same solution

and in the latter case a shall pattern which supports the specification of search problems.

Class and concept creation If similar shall patterns $\left(\mathrm{s}^{\mathrm{A}}, \mathrm{s}^{\mathrm{B}}\right)$ are found and combined ( $\mathrm{s}^{\mathrm{A}} \sim \mathrm{s}^{\mathrm{B}} \rightarrow \mathrm{s}^{*}$ ), the result obtained is a reusable shall class. A similar result applies to

- design patterns $\left(\mathrm{d}^{\mathrm{A}} \sim \mathrm{d}^{\mathrm{B}} \rightarrow \mathrm{d}^{*}\right)$ and

- test patterns $\left(t^{A} \sim t^{B} \rightarrow t^{*}\right)$.

\section{Classes}

Class of shalls

$\mathrm{s}^{\mathrm{A}} \sim \mathrm{s}^{\mathrm{B}} \rightarrow \mathrm{s}^{*}$

O

$$
\begin{array}{cc}
0 & O \\
t^{A} \sim t^{B} \rightarrow t^{*} & d^{A} \sim d^{B} \rightarrow d^{*} \\
\text { Class of tests } & \text { Class of designs }
\end{array}
$$

$t^{i}$ is test $t$ of project $i ; s{ }^{i}$ is shall $s$ of project $i ; d^{i}$ is design $d$ of project $i ; \rightarrow$ is a generalization

Figure 16 Generalization concept I

Naturally, it is possible to generalize not only links between models of the same type but also links between models of different types. A design concept is thus obtained if it is possible to generalize links between shall classes and design classes

- $\left(\mathrm{s}^{*} \rightarrow{ }^{\mathrm{A}} \mathrm{d}^{*} \& \mathrm{~s}^{*} \rightarrow \rightarrow^{\mathrm{B}} \mathrm{d}^{*} \Longrightarrow \mathrm{s}^{*} \rightarrow^{*} \mathrm{~d}^{*}\right)$.

A design concept then answers the question as to which idea can be implemented 
Design concepts

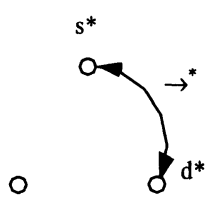

Test concepts

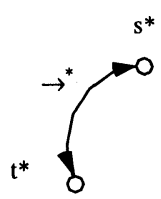

Development concepts

$\circ$

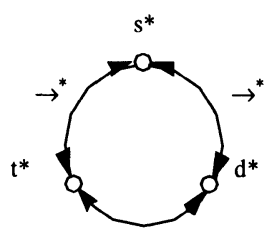

$\mathrm{t}^{\mathrm{i}}$ is test $t$ of project $\mathrm{i}$; $\mathrm{s}^{\mathrm{i}}$ is shall $\mathrm{s}$ of project $\mathrm{i}$; $\mathrm{d}$ is design $\mathrm{d}$ of project $\mathrm{i} ; \rightarrow^{*}$ is a generalized link

Figure 17 Generalization concept II

and how. Which idea can be validated/verified and how is defined by means of a test concept. This is produced through

$$
\text { - }\left(\mathrm{s}^{*} \rightarrow^{\mathrm{A}} \mathrm{t}^{*} \& \mathrm{~s}^{*} \rightarrow^{\mathrm{B}} \mathrm{t}^{*} \Longrightarrow \mathrm{s}^{*} \rightarrow^{*} \mathrm{t}^{*}\right) \text {. }
$$

It is of course also conceivable that design classes and also test classes correlate with a shall class. Only in this situation are tests which are constructed exclusively via shalls (design independence; designs should be checked regarding their consistency with their shalls) linked implicitly with the design.

Finally, the SDT development model is represented in overview from the reuse perspective (Figure 18).

The upper section of the Figure 18 shows the current project. It is now possible during the SDT partitioning process to refer back to experience gained in the description of shalls, designs or tests from older projects (projects A, B). This can be done on a per project basis on the one hand or by way of generalized project experience on the other. The consequences of reusing a component from an earlier project can now be examined by way of their description in the shalls both for the current and also for the following abstraction steps. It is similarly possible to look ahead to realizations of later abstraction steps. Such considerations help to more accurately define the objective of a development.

\section{SUMMARY AND OUTLOOK}

The object of this paper was to present a model which takes a new look at the development process for a product through parallelization of specification, design and testing. Taking an analysis of proven development models as the starting point, the conclusion was an information model which organizes the specification problem in a Shall Model, the design problem in a Design Model and the verification/validation problem in a Test Model, and which describes relations linking the various models. The information model presented makes it possible to consider the consequences of a partial redesign and to describe the reuse and also the generalization of project knowledge in the form of shalls, designs and tests.

It will be the object of future work to formulate a shall, design and test classifi- 


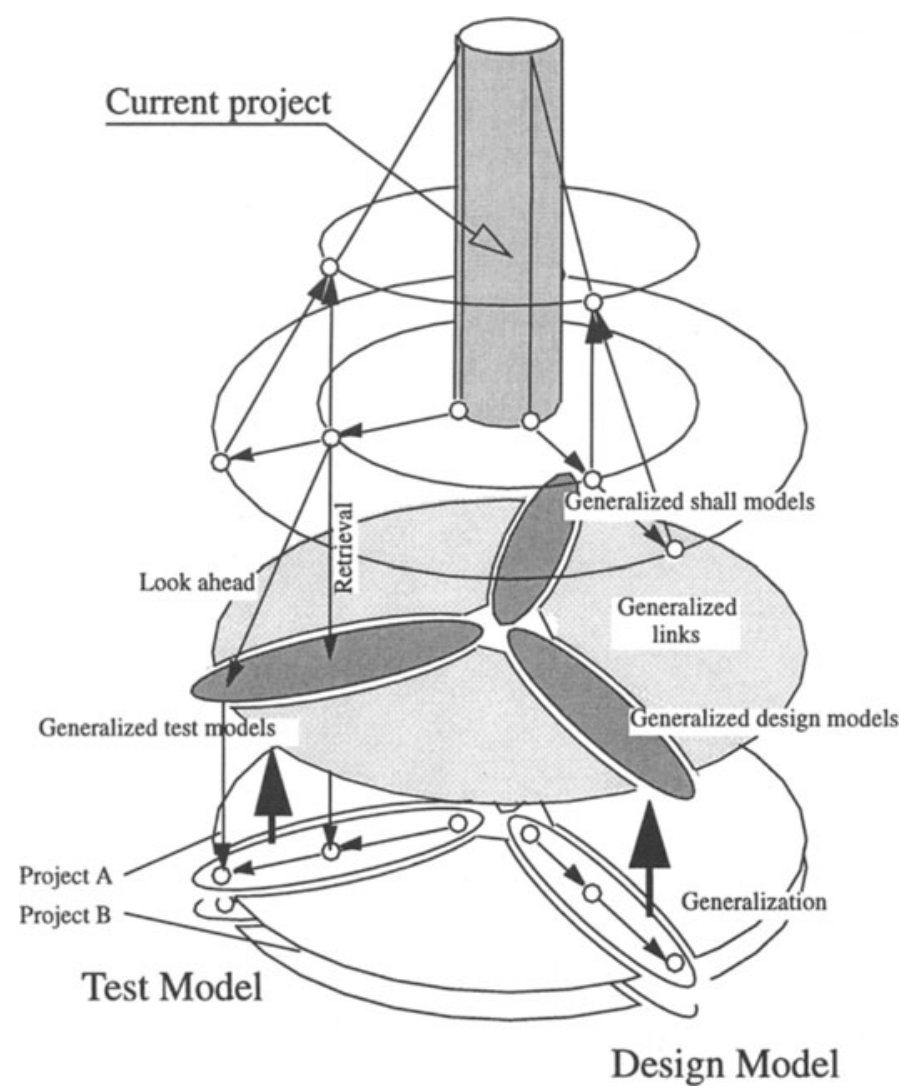

Figure 18 The SDT development model from the reuse perspective

cation, and also to draw up typical design concepts (assignment of shalls to designs shall-design pattern) as well as testing concepts (assignment of shalls to tests shalltest pattern).

\section{REFERENCES}

Bauer, Böttger, Ecker, Jensen(1996) Modeling Monitors in VHDL. VHDL International Users' Forum (VIUF'96 Fall).

Beck(1993) CRC: finding objects the easy way. Object Magazine. Vol.3, No.4.

Boehm(1980) Software Economics. Prentice Hall.

Boehm(1984) Verifying and Validating Software Requirements and Design Specifcations. IEEE Software.

Boehm(1986 (August)) A Spiral Model of Software Development and Enhancement. ACM SEN 11, 3. 
Booch, Rumbaugh(1996) Unified Method 0.8. http://www.rational.com.

Burns, Wellings(1995) HRT-HOOD: A Structured Design Method for Hard RealTime Ada Systems. Elsevier.

Camurati et al(1993) A methodology for system-level design for verifiability. Correct Hardware Design and Verification Methods.

Coleman et al(1993) FUSION: A second generation object-oriented analysis and design method. Object EXPO Europe Conference.

Cyre, Amstrong, Honcharik(1996) Generating Simulation Models from Natural Language Specifications. Simulation, Vol. 65, No. 4.

Davis(1990) Software Requirements. Analysis and Specification. Eng.wood Cliffs. Ecker(1995) A Classification of Design Steps and their Verifications. Euro-DAC'95. Flor(1996) Konfiguration und Management wiederverwendbarer Softwarekomponenten. Component User's Conference.

Fritzsch(1995) Compiler für die Übersetzung von ESCs in VHDL- und C-Code. Diss. for Diploma, Technical University of Chemnitz-Zwickau, Dept. Computer Science, 09107 Chemnitz, Germany.

Gajski(1994) Specification and Design of Embedded Systems. Prentice Hall.

Kahlert et al(1993) High-level Entwurf und Analyse mit Erweiterten Sequence Charts (ESC). Proc. 6. EIS-Workshop.

Hein et al(1996) RASSP VHDL Modeling Terminology and Taxonomy. http://rassp.scra.org.

Hodgson(1991) The X-Model: A Process Model for Object-Oriented Software Development. Proc. 4th Int. Conf. Software Engineering and its Application.

IEEE Computer Society(1993) IEEE Recommended Practice for Software Requirements Specifications. IEEE Std. 830-1993.

Jacobson et al (1995) The Object Advantage. Business Process Reengineering with Object Technology, Addison-Wesley.

Mrva, Stal(1997) Die veränderte Rolle des Software-Designers. to appear in Informatik-Spektrum.

Preis(1996) Making Reuse happen in the Design Process. SIG-VHDL Spring '96 Working Conference.

Sahraoui, Six, Bolsens, DeMan (1995) Search Space Reduction Through Clustering in Test Generation. Euro-DAC'95.

SIA National Technology Roadmap for Semiconductors - 1994(1995) MP.Brassington. IEEE 1995 CICC, 0-7803-2584-2/95.

Siegel, Eichele(1990) Hardwareentwicklung mit ASIC. Heidelberg: Hüthig Buch.

Shlear, Mellor(1996) The Shlear-Mellor Method. http://www.projtech.com .

Vemuri, Kalyanaraman(1995) Generation of Design Verification Tests from Behavioural VHDL Programs Using Path Enumeration and Constraint Programming. IEEE Transactions on VLSystems.

Northern Telcom(1996) VHDL in Use: Experiences from Telecom and Networking. Panel, VHDL International Users ' Forum (VIUF'96-Spring).

Waxman(1995) The Union of Specification Modeling with the Design Process. VHForum for $\mathrm{CAD}$ in Europe. 